\title{
El Estudio General de la Orden de Predicadores y la Universidad Tomística de Estudios Generales en la Nueva Granada
}

Édgar Arturo Ramirez Barreto*

E ste capítulo quiere precisar diferencias y similitudes, en la Nueva Granada, entre el Estudio General de la Orden de Predicadores con los Estudios Generales de la Universidad Tomística de Santafé de Bogotá ${ }^{1}$ Y, por otra parte, comparar el carácter universitario de la Tomística de Santafé frente a algunas universidades en la América del periodo colonial hispánico.

* Filósofo, Universidad Nacional de Colombia; magíster en Historia y Teoría del Arte, la Arquitectura y la Ciudad, Universidad Nacional de Colombia. Docente del Departamento de Humanidades y Formación Integral, Universidad Santo Tomás, Bogotá.

1 En la bula de 1580, dada por "su Santidad Gregorio XIII, por medio de la cual se aprueban los privilegios concedidos en el Capítulo General a la Provincia de San Antonino de la Orden de Santo Domingo en Santa Fe de Bogotá y se erigen en universidad su convento de la virgen del Rosario", se otorga el privilegio de "vnam Universitatem Studij generalis..." (Hernádez de Alba, 1969, pp. 33-34). 
Para el desarrollo de esta problemática se intentará definir el concepto de Estudio General a través de la exploración del documento "De las constituciones, Declaraciones y Ordenanzas, Capítulo General", el cual trata de los estudios en los primeros siglos de la Orden de Predicadores en Europa, y también en el texto Rationes estudiorum, que se encuentran en la obra Compendio de historia de la Orden de Predicadores ${ }^{3}$; para luego señalar la relación que existió en el siglo XVI en España entre los Estudios Generales al interior de la Orden de Predicadores y las primeras universidades españolas, en especial la de Salamanca. Esto con el fin de proponer una distinción entre el Estudio General conventual y los Estudios Generales universitarios en la Tomística de Santafé de Bogotá en el periodo neogranadino.

La Universidad Tomística se estableció en el seno de la Orden de Predicadores en la denominada, para la época, Provincia de San Antonino ${ }^{4}$. Esta provincia fue fundada en 1550, la cuarta en América en orden cronológico ${ }^{5}$, al tiempo que se instauraba en Santa Fe de Bogotá la Real Audiencia (Plata Quezada, 2012, p. 49)

2 De las constituciones, Declaraciones y Ordenanzas, Capítulo General, dadas desde el año 1220 hasta el año 1659, publicadas y organizadas por el reverendísimo padre fray Vicente Fontana y divulgadas en 1655, continuadas hasta 1862, a ellas se añade, según el parecer del Capítulo General de Roma en 1811 ordenación tercera, muchos decretos más recientes de las Constituciones Apostólicas y la Sagrada Congregación. Obra compilada y editada por el reverendo padre maestro hermano Cajetano Lo-Cicero de la Provincia Patrinacria, bajo la dirección del reverendísimo padre maestro de la Orden Alexandri Vicentii Jandel. Primera parte, Roma. Tipografía Bernardi Morini, 1862. Traducción libre del Documento Original en latín, a cargo de Dayan Herrera Bernal.

3 Compendio de historia de la Orden de Predicadores, § 38. Rationes studiorum. Traducción libre, del latín al español, a cargo de Dayan Herrera Bernal.

4 Actualmente, la Provincia de la Orden de Predicadores se denomina San Luís Bertrán de Colombia.

5 La primera provincia fundada por los dominicos en América fue la novohispana de Santa Cruz de las Indias en 1530, luego la provincia de Santiago de 


\section{Los Estudios Generales en la Orden de Predicadores}

De acuerdo con "las constituciones, declaraciones y ordenanzas, Capítulo General", en el título "Sobre los Estudios Generales", numeral 1., en principio los dominicos solo podían llevar a cabo el Estudio General en París. Pero, en razón al aumento de los estudiantes, el Capítulo General decidió que a partir de 1302 se podía impartir Estudio General en algún "convento idóneo" de ciertas provincias europeas de la época. Tales como la provincia de Provenza, la de Lombardía, Alemania e Inglaterra; y en Bolonia se aprobó que también se instituyeran en las provincias de España y Roma, lo que conllevaría, hacia el futuro, que en cada provincia europea se debía, entonces, erigir un Estudio General.

Ahora, en cuanto a los contenidos del Estudio General, cabe señalar lo siguiente:

En los Estudios Generales no solo se enseñan los Tratados Especulativos sino también, los Morales. Bolonia 1564. Ord. 4. Además en los Estudios Generales deben tenerse siempre dos clases sobre las sentencias o partes de Santo Tomás y no solo lo Especulativo sino también lo Moral debe ser enseñado por los lectores y que no se cambie nada so pretexto de enseñar la Sagrada Escritura sobre la cual se habló en relación con

México en 1532; en 1540 la Provincia del Perú, y la Provincia de San Antonino del Nuevo Reino de Granada en 1850 (Plata Quezada, 2012, p. 49).

6 De las constituciones, Declaraciones y Ordenanzas, Capítulo General...

7 De las constituciones, Declaraciones y Ordenanzas, Capítulo General... "Desde la Constitución Nuestra distinción 2. Cap. 14 texto 3. Que fue dada en Bolonia en 1302. Confirmada en Bisuntia en 1303, y en la Constitución redactada en Tolosa en 1304". 
los Estudios Escolásticos de la Teología. Pero según la Carta Encíclica del Reverendísimo Maestro de la Orden Jandel el día 14 de julio 1852 dicta. Ordenación 4. Del Capítulo General de Bolonia 1546. Tuvo una modificación. Véase. Núm. 15. Sobre los estudios. Ver muchas cosas relativas a la materia sobre los Regentes, Bachilleres, Maestros de estudios, exámenes de los Hermanos y grados.

A partir de lo anterior se puede decir, por ahora, que a comienzos del XVI el Estudio General de los dominicos es el estudio especializado sobre Santo Tomás en lo concerniente a los tratados especulativos y morales; y también que estos estudios se realizaban en París durante los comienzos de la Orden y que, posteriormente, el Estudio General paulatinamente se trasladó a las distintas provincias. Así pues, se esperaría que cada provincia dominica tuviese estos estudios, al menos, en alguno de sus conventos.

Esto se corrobora, y se precisa mejor, en el Compendio de historia de la Orden de Predicadores, en lo referente a Rationes studiorum ${ }^{8}$. Allí se estipula, en el plan de estudios de las constituciones de 1228, "que la provincia envíe al estudio en París solamente tres frailes". "Cuando de una provincia se envía a otra un fraile para dirigirla debe llevar consigo todos los libros, glosas, la Biblia y los cuadernos".

$\mathrm{Y}$ en cuanto al contenido de los estudios se sostiene:

Al comenzar el siglo XIV en Génova (1305) y en Venecia (1325) se ordenaron cosas importantes en relación con los estudios. Pues "nadie notoriamente joven sea enviado a los estudios de arte antes de tres años de haber ingresado". Después viene el

8 Compendio de historia de la Orden de Predicadores, § 38. Rationes studiorum...

9 Ibídem. Pág. 1 de 11. 
estudio de la lógica durante tres años de los temas naturales durante dos años de la teología durante dos años. Para los lectores estuvo vigente un aprendizaje aún más severo en relación con estos estudios, ya que nadie podía estudiar temas lógicos sin mostrar adelanto en temas naturales durante dos años ni enseñar temas naturales sin aprovechamiento durante dos años en teología ni enseñar teología en un estudio general sin haber cursado dos años de teología en un estudio especial. Por consiguiente, en general a partir de mediados del siglo xiır hay en todas partes estudios de artes, o sea lógica. Estudios de temas naturales o sea física, estudios de teología ${ }^{10}$.

De esta cita se puede deducir que por Estudio General se entiende lo concerniente a la Teología, lo que en "las constituciones, declaraciones y ordenanzas, Capítulo General" se toma como el estudio especializado de Santo Tomás.

Con la siguiente cita, tal vez se tenga una idea más amplia y completa del contenido de los estudios de la Orden de Predicadores en Europa alrededor de los siglos XIV y XV:

Para los que debían dedicarse a los estudios una vez cumplido cabalmente el noviciado se requerían nociones gramaticales y de latín. Después debían aprenderse las artes, o sea, la filosofía y finalmente la teología cada vez más según la suma del Aquinate. Así después de los capítulos anteriores en Roma en el año 1580 se establecieron un trienio de filosofía (que comprendía dialéctica, filosofía y metafísica) y un cuatrenio de teología sobre la Suma de Santo Tomás, mientras en el plan de estudios bajo el reverendísimo Sixto Fabri en el año 1585 "con el parecer de los dignísimos padres" se atribuyen también dos años de

10 Ibídem. 
lógica, tres años de filosofía, es decir, de física y metafísica, de teología para la suma partes I y II de la Suma en cuanto al tema "especulativo" para la parte I de la primer y II de la segunda en cuanto a la doctrina moral se asignan cuatro años ${ }^{11}$.

A partir de la anterior descripción de los estudios de los frailes dominicos en Europa conviene ahora ver cómo se traducían estos, en específico, a la España del siglo xvi:

En los primeros capítulos provinciales [de los Dominicos] del siglo XVI se sintió el impulso dado al estudio por los generales de la Orden Vicente Bandelli (1501-1506) y Tomás de Vio Calletano (1508-1518). En jerez (1502) y en Peñafiel (1504) — este último celebrado en presencia del Maestro General- se insistió en la importancia de los estudios y se planteó la necesidad de un acuerdo provincial que posibilitara el envío de los mejores estudiantes de cada convento a los Estudios Generales. (Ramírez González, 2001, p. 148)

La principal característica de los estudios dominicos en la España del siglo XVI es que "al mismo tiempo que se intentaban estructurar los estudios dentro de la comunidad, se buscaba su proyección hacia las universidades" (Ramírez González, 2001, p. 149). De tal manera que "la Universidad de Salamanca aceptaba los cursos conventuales de artes como equivalentes al grado de bachiller en artes y consideraba algunos otros cursos y los años de práctica dentro de la orden como válidos para los grados en teología que ella otorgaba" (Ramírez González, 2001, p. 150).

En España, hay también que destacar que, al rastrear los estudios de los dominicos dentro de la Orden de Predicadores, se

11 Ibídem. b) Vita et Actio Scientifica. § 78. Rationes estudiorum. Pág. 9 de 11. 
manifiesta que no eran estudios que el alumno llevara a cabo en un mismo convento, sino que los estudios se distribuían por toda la península Ibérica. Además, cabe precisar que no todos los conventos de la Orden eran idóneos para el Estudio General. La autora señala la complejidad y la dificultad para seguir la estructura de esta formación; sin embargo, ella misma presenta como ejemplo la posible formación en una Provincia:

La legislación de estos primeros años del siglo refleja, pues, el esfuerzo de los dominicos por integrar y estructurar una red de estudios para la provincia de Castilla. En 1511, en el Capítulo Provincial de Sevilla, se resumió tal sistema de estudios.

En primer lugar, se recordó que para tomar el hábito son obligatorios los conocimientos elementales de gramática latina, y a continuación, se fijó la jerarquía académica asignada a los diversos conventos de la provincia:

Conventos dedicados al perfeccionamiento de la gramática: Villada (Palencia), Carboneras (Cuenca), Burgos, San Pedro de Dueñas (Segovia), Peñafiel (Valladolid), Écija y Benavente.

Conventos dedicados a la lógica y las artes: Córdoba, Toledo, Santa María Real de Nieva, Segovia, Toro y Palencia.

Estudios generales: San Esteban de Salamanca, San Pablo de Valladolid, San Pablo de Sevilla y Santo Tomás de Ávila. (Ramírez González, 2001, p. 150)

Para la época, también existía otro tipo de establecimiento de educación: los colegios, que eran anexos a muchos conventos, pero que a veces tenían cierta independencia económica e institucional. Aquí también la autora destaca la dificultad de entender su función dentro del entramado de la formación y de la expedición de títulos.

Si se compara el colegio dominico de San Gregorio de Valladolid con el de Santo Tomás de Sevilla, puede verse cómo dos 
instituciones similares en su concepción tuvieron desarrollos distintos. San Gregorio se conservó como colegio exclusivo para la perfección de los estudiantes dominicos, mientras que Santo Tomás se abrió hacia otros estudiantes y buscó su reconocimiento como universidad. (Ramírez González, 2001, p. 153) ${ }^{12}$

En cuanto a los conventos, el Estudio General que más prestigio tuvo en España fue el del convento dominicano de San Esteban de Salamanca, sobre todo en la época en que Francisco de Vitoria fue regente de estudios del mismo claustro, y catedrático de prima en teología de la Universidad de esta misma ciudad. En San esteban el programa de estudios durante este periodo comprendía gramática, lógica, filosofía y teología:

Porque la diversidad de opiniones suele engendrar la máxima confusión, ordenamos y mandamos que en los conventos de nuestra congregación no se lea otro arte de gramática que el de Nebrija.

Para la filosofía se usaba, en principio las Súmulas de Pedro Hispano, y los libros de Aristóteles, pero la presencia en el convento de Domingo de Soto propició que se adoptaran sus obras en el Estudio hacia mediados del siglo xvi. [...]

En teología, Vitoria reforzó la importancia de la Summa de Santo Tomás, sobre las sentencias de Pedro Abelardo. [...]

12 Esta compleja relación entre universidad, convento y colegio en el ámbito de los dominicos la vemos en la Nueva Granada a finales del siglo XVI y comienzos del Xvir entre el Convento de Nuestra Señora del Rosario de Santafé de Bogotá, la Universidad Tomística, el Colegio de Santo Tomás y el Colegio Mayor de Nuestra Señora del Rosario. De paso, hay que decir que este trabajo se restringe a la educación de los dominicos exclusivamente en Santafé de Bogotá, y que tampoco se tendrá en cuenta las instituciones educativas de los jesuitas, o de los agustinos, por ejemplo, que tan influyentes fueron durante el mismo periodo en esta ciudad. 
En cuanto al tiempo de lectura para cada materia, el mismo capítulo [Capítulo General de Cataluña 1574] resumía el sistema de estudios dominico de la siguiente manera "Confírmase que oyesen los estudiantes siete años, tres de artes y cuatro de teología, y que los dos lectores de teología se conumasen de manera que el uno leyese materias especulativas y el otro morales. (Ramírez González, 2001, p. 141)

La relación de los teólogos del Convento de San Esteban con los profesores de teología de la Universidad de Salamanca explica también la intención de los frailes dominicos frente al nuevo fenómeno educativo e institucional de las universidades en España, que era la de estar presente en la universidad a través de la facultad de teología puesto que el título de teólogo o el reconocimiento como tal solo podría ser expedido, y hacia el futuro, por la universidad y no tanto por los estudios conventuales.

\section{Los Estudios Generales conventuales frente a la Universidad}

La explicación que ofrece Juan Belda (Belda Plans, 2000) acerca de por qué París dejó de ser el centro de los Estudios Generales en Europa es que antes del Gran Cisma de Occidente dicha ciudad era la única que contaba con una universidad con estudios en teología; y Bolonia, por su parte, con la única universidad jurídica. Lo anterior le permitía al Papado un eficiente control de sus doctrinas en estos centros de formación, pero con el Cisma de Occidente la Santa Sede vio la necesidad de comenzar a multiplicar los estudios de teología en toda Europa. En consecuencia, los Estudios Generales terminaron siendo impartidos por los conventos, al menos lo fue en España, y específicamente en Salamanca, a través del convento dominicano de San Esteban. 
Según este autor, Salamanca fue "considerada como uno de los cuatro principales Estudios Generales de la cristiandad (junto con París, Bolonia y Oxford)" (Belda Plans, 2000, p. 124). Pero los estudios teológicos, como Estudios Generales, no se realizaron en la Universidad, sino en el convento dominico de San Esteban.

Pese a lo anterior se afirma que:

El Estudio General de San Esteban se revela como un conjunto complejo. Acogía en su seno estudiantes de otros conventos e incluso de otras provincias, además de los del propio convento, a los que organizaba de muy distintas maneras: colegios o regímenes especiales. Tenía lecciones en su interior, pero también permitía que sus oyentes asistieran a las lecciones de la Universidad y participaran en sus actos públicos, y a la vez permitía que alumnos externos oyeran algunas lecciones en el convento. Pero, además formaba parte de una red de estudio común a la Orden de Predicadores y quedaba ligado a la Universidad a través de sus cátedras (Ramírez González, 2001, p. 146)

El Estudio General de San Esteban quedaba, pues, vinculado a la Universidad a través de las cátedras de Teología. Pero, además de los vínculos que establecían los oyentes, la relación se daba a través de los catedráticos. Ellos tenían que incorporar sus grados a la Universidad, hecho por el cual pasaban a ser sus miembros: el grado les daba participación en los claustros plenos y de diputados (principales órganos de gobierno de la corporación) y los hacía beneficiarios de sus rentas. (Ramírez González, 2001, p. 145)

Ahora bien, ¿cuál sería, para entonces, la diferencia entre los estudios de una universidad como la de Salamanca frente a los Estudios Generales en los conventos? En cuanto a la organización de los estudios de la Universidad de Salamanca, se sostiene: 
La universidad salmantina constaba de cuatro Facultades Mayores, a saber: Cánones (o Derecho Canónico); Leyes (o Derecho Civil); Teología y Medicina. Además, estaba la Facultad de Artes (o Filosofía), que era considerada Facultad Menor. Para estudiar Teología o Medicina era necesario haber estudiado Artes con anterioridad. Los títulos que se podía obtener es todas estas Facultades eran los de Bachiller, Licenciado y Doctor (o Maestro).

La facultad de Teología tenía tres cátedras principales llamadas Mayores (o también de propiedad o vitalicias): La de Prima (a primera hora de la mañana) y la de Visperas: (a primera hora de la tarde), donde se leía el Maestro de las Sentencias de manera orgánica; las clases duraban una hora y media en la Prima, y una hora en la de Visperas. Además, estaba la cátedra de $B i$ blia, a la hora de tercia, en la que se explicaba alternativamente un año el Antiguo y otro el Nuevo Testamento. Estas cátedras constituían la base de la enseñanza teológica, eran vitalicias y estaban bien pagadas, tenían profesores sustitutos y a los veinte años de docencia había derecho de jubilación. [...]

Se añadían todavía otras tres llamadas cátedras menores o catedrillas: la de Santo Tomás; de Escoto; y de Nominales (más tarde de Durando); al principio del siglo xvi las dos primeras, que ya existían antes en los respectivos Conventos de Mendicantes, se trasladan a la Universidad. (Belda Plans, 2000, p. 127)

En el título anterior, que se refiere a los estudios conventuales de los dominicos, se relacionan los estudios conventuales de San Esteban. Si se comparan estos estudios con los estudios universitarios, considero que la Facultad de Teología subsume los Estudios Generales que se impartían en el convento. Por lo tanto, si quisiéramos hacer una diferencia entre los estudios universitarios y los conventuales, se podría decir que los estudios conventuales son los denominados Estudios Generales (en los dominicos), y estos mismos estudios, a mi 
modo de ver, en la universidad, son los concernientes a la Facultad de Teología. De esta manera, se puede afirmar que, al imponerse progresivamente los estudios universitarios como legítimos estudios reconocidos por las instituciones estatales, el reconocimiento de los títulos universitarios va exigiendo a los conventuales el traslado del convento a la universidad, al menos, lo concerniente a la teología y su titulación.

Teniendo en cuenta lo anterior, ¿qué era, entonces, una universidad? Si, tal como se ha expuesto, los Estudios Generales eran estudios teológicos propios del carácter de un convento; a la universidad, entonces, la caracterizaría el contar con las distintas facultades, así como el hecho de que no sean instituciones necesariamente clericales, sino estatales, o mejor monárquicas, puesto que la monarquía era el sistema político del Imperio español de la época a la que referimos.

\section{Las universidades en la América hispánica}

El Imperio español procuró que las universidades que se fundasen en las indias I Filipinas siguiesen el modelo de la Universidad de Salamanca. Y se puede decir que, al menos, en América fueron dos las universidades las que cumplieron con este dictamen: la Universidad de San Marcos de Lima y la Real Pontificia Universidad de Méjico.

La Universidad de San Marcos de Lima tuvo unos comienzos un tanto agitados, como consecuencia de la situación sociopolítica conflictiva, que hizo retrasarse el comienzo regular de sus actividades. En su origen estuvo ligada al convento limense del Rosario de los Dominicos, que contaba con estudios de Artes, Teología y Sagrada Escritura, siendo su principal impulsor fray Tomás de San Martín, O. P., quien solicitó a Carlos V, 
en nombre de la Orden y de la ciudad de Lima, Fundar en el Convento Dominicano unos Estudios Generales con los mismos privilegios que la Universidad de Salamanca. Una vez puesta en marcha en 1551, surgieron conflictos con las autoridades civiles que querían independizarla de los dominicos; el contencioso dura hasta 1571 en que se produce la separación por obra del virrey Toledo, comenzando así un nuevo periodo laico de la Universidad limense.

La Realy Pontificia Universidad de Méjico tiene una larga historia de peticiones por parte de las autoridades civiles y religiosas. Durante las primeras décadas del siglo Xvi el terreno se fue abonado por los colegios y Estudios Superiores de las distintas Órdenes Religiosas, cuyos integrantes en su mayoría tenían procedencia universitaria salmantina. Quien comienza a dar pasos definitivos es el obispo fray Juan de Zumárraga, ofM, quien hacia 1538 pide a Francisco de Vitoria en Salamanca le envíe letrados de buena vida, cosa que se hace oficial cuando Carlos V secunda los deseos del obispo mejicano, encarga en 1539 a Vitoria que seleccione a doce discípulos suyos para mandarlos a Nueva España. El inquieto Zumárraga intenta incluso traer a Domingo de Soto para que resuelva los numerosos problemas teológicos y morales que se suscitaban por entonces allí. La petición de una Universidad pronto fue unánime por parte de todas las fuerzas vivas. Finalmente se crea la Universidad, como vimos, a finales de 1551 conforme la modelo de Salamanca y con todos sus privilegios.

En su origen constaba de cuatro Facultades con un total de nueve cátedras. Teología: cátedras de Prima, Santo Tomás (Visperas), Sagrada Escritura; Derecho Canónigo: cátedra de Cánones; Derecho Civil (Leyes): cátedras de Derecho Civil y de Instituciones; Filosofía cátedras de Artes, Retórica y Gramática. Eran vitalicias (así las de Teología y Prima y Visperas) o temporales (por cuatrienios), y, como en Salamanca, todas las cátedras se 
ganaban por oposición pública con intervención del alumnado.

(Belda Plans, 2000, p. 873)

Con la realidad que se estaba presentando se podían evidenciar la existencia de dos modelos posibles de universidad en América. Por un lado, el de la universidad pública, que fue el caso de México; y, por otro, el de la universidad perteneciente a una orden regular, que fue el caso de la universidad dominicana de Lima, en su primera etapa. La universidad mexicana era fiel copia de la Universidad de Salamanca; mientras que la de Lima era émula de las universidades españolas dominicanas como la de Ávila o Pamplona; de tal modo que el cargo de rector, en el periodo dominicano de la Universidad de San Marcos de Lima, lo ejercía el prior del convento (Rodríguez Cruz, 1973, p. 192); en tanto que el primer rector de la Real y Pontificia Universidad de Méjico fue el oidor don Antonio Rodríguez de Quesada, quien fue nombrado por el virrey Velazco (Rodríguez Cruz, 1973, p. 250). Pero la laicización definitiva, o el paso de institución religiosa a institución virreinal o del Estado, de la Universidad de San Marcos de Lima solo ocurre hasta 1571:

El claustro universitario, una vez independizado del convento de los dominicos, elabora las primeras constituciones conocidas que tuvo la limeña, por encargo especial del virrey Toledo. Compuestas por el doctor Pedro Fernández de Valenzuela, primer rector seglar, fueron la base de las que se redactaron después y en ellas se descubre la huella salmantina. Consta de cuarenta y tres estatutos. Versan acerca de la elección del rector, anualmente, por votación secreta del claustro, que elegía también dos consiliarios. El rector debía ser laico y estaba obligado a velar por la buena marcha de la Universidad, convocar el claustro para estudiar los problemas universitarios, con cierta periodicidad, cuidar de que los catedráticos cumplieran con 
su deber y de que los estudiantes se matricularan para que sus cursos tuvieran validez. (Rodríguez Cruz, 1973, pp. 196-197)

Estas dos universidades contaron con sus respectivas facultades y con la legitimidad real y eclesiástica para la expedición de títulos, además, ambas en su momento de esplendor fueron públicas tal como lo era su alma mater, la de Salamanca. Pero estas dos universidades, una en México y la otra en Perú, no fueron suficientes para satisfacer las necesidades educativas en las demás regiones de la vasta colonización de los españoles en América, razón por la cual se permitió la fundación de otros centros de estudios universitarios en otras capitales. De este modo, a los centros universitarios en América se les dividió en dos categorías: "las dos oficiales de Lima y México, a las que llama generales o de estudios generales", y "las menores, de cátedras y privilegios limitados, con facultades restringidas para graduar [...] que van surgiendo teniendo generalmente como base una fundación conventual o colegial, sobre todo de dominicos y jesuitas" (Rodríguez Cruz, 1973, p. 7)

Pese a lo anterior, permanece la duda sobre qué se entiende por Estudios Generales, sobre todo cuando se habla de universidades en su sentido pleno.

Para dar respuesta a la cuestión mencionada, se hará alusión a una nota del agustino José Abel Salazar, citado a su vez por Águeda Rodríguez, O.P., en el siguiente pie de página aclaratorio:

Para José Abel Salazar la división que da la Recopilación de Indias sobre universidades 'generales' y 'particulares' podría entenderse tomando las primeras en el sentido de 'públicas' o 'estatales', o sea gobernadas con intervención de la autoridad regia, sometidas inmediatamente a su patronato, erigidas independientemente de otra entidad moral que estorbara las actuaciones del patronato, y sostenidas o con fondos del real erario o con otros ofrecidos por particulares, pero ya secularizados 
y puestos bajo arbitrio del monarca, como en Lima y México. También incluye, aunque apurando el criterio, a las de Caracas, Córdoba (en los finales del periodo hispánico) y San Felipe de Santiago de Chile (los estudios eclesiásticos superiores en el Nuevo Reino de Granada [...]). El sentido 'público' como equivalente a 'estatal' atribuido al término 'general' aplicado a las universidades, como opuesto a 'particular', pareció que fue el que prevaleció entre los tratadistas de la época, entre ellos, el mismo consejo de Indias, el más auténtico intérprete de las leyes. Según este criterio, las universidades 'generales' o 'públicas' eran las fundadas como tales por el Papa y confirmadas por el Rey, sin que la aprobación real, o sea el pase regio, fuera necesaria para su entidad o existencia, aunque sí para el ejercicio de sus funciones cuando avanzada la dominación española se acentuó el regalismo e intervencionismo del estado y se fue ampliando el alcance del real patronato, sobre todo a partir del reinado de Felipe II, lo que no ocurrió en la primera etapa civilizadora. A veces la erección la hizo primero un documento real para la que luego se pidió la confirmación pontificia. Las universidades particulares eran las que tenían el mero privilegio de graduar en sus estudios privados, generalmente en el seno de una comunidad o colegio. Para algunos, el carácter 'público' de algunas universidades se refiere a haber sido fundadas con autoridad real, distribuyéndolas sin embargo en dos categorías, 'mayores' y 'menores' atribuyendo la primera categoría a las que la Recopilación llama 'generales' y la segunda a las 'particulares'. Pero hay que tener muy en cuenta que las universidades pontificias también son públicas, en cuanto que han sido erigidas por una autoridad pública, el papa, y con facultad para ello, según las partidas (partida II tít. xxxi, ley $1^{\circ}$, edición de la Real Academia de Historia, Madrid, 1807). Y todas las llamadas 'particulares' también fueron 'generales' en cuanto que las fundó el rey o el papa y por lo menos enseñaron 
artes y teología, según la misma legislación de las Partidas. (Rodríguez Cruz, 1973, p. 28)

Se puede concluir así que una Universidad de Estudios Generales era una universidad pública, regia o perteneciente a la monarquía. Más que todo, a partir de Felipe II, quien acentuó el Patronato Regio, o la administración de las funciones de la institución de la Iglesia española bajo el dominio de la monarquía. Esto es, que el papado de Roma fue perdiendo la injerencia exclusiva sobre las jerarquías eclesiásticas en el Imperio español, ya que la majestad del rey le daba la potestad de nombrar obispos y miembros de la iglesia en España, bajo aprobación del papa, en virtud al acuerdo del Patronato Regio con el Vaticano (Calderón, 2010). En consecuencia, la función educativa pasaba de las manos de la Iglesia, a las del Estado. Y con respecto a las universidades en la América hispánica, esta tendencia se vio especialmente en los Virreinatos de la Nueva España y Perú.

\section{Los estudios en el Convento Dominicano de la Virgen del Rosario en Santafé de Bogotá}

Según Fray Alonso de Zamora, O. P. (Zamora, 1945, p. 229), los primeros estudios en el convento del Rosario comenzaron alrededor de 1563, con las lecciones de gramática:

Con diez, y siete Conventos se hallaba la Provincia al tiempo de su erección, y todos fueron admitidos con titulo de Prioratos; hallábase también con mas de cien Religiosos, los mas, que avian venido de España en las misiones, que llevo referidas; y algunos, que avian tomado del Abito, y professado en este Convento del Rosario, y en los de Cartagena y Tunja, desde el año de 1550, que se fundó la Congragacion. 
Hallavanse quatro Maestros, el P. Provincial Fr. Francisco Vanegas, el P. Fr. Antonio de la Peña, el P. Fr. Alberto Pedrero, y el P. Fr. Pedro Martir Palomino. Quatro Presentados, el P. Fr. Bartholomé de Ojeda, el Fr. Juan de Monte-mayor, el P. Fr. Antonio de Miranda, y el P. Fr. Luis Lopez.

Fue electo el Provincia el M. R. P. Presentado Fr. Antonio de Miranda, de Nación Lusitano, hijo de la Provincia de Portugal. Vino á esta, segun parece el año 1552. Porque el de 1555. Se halla, que se le repartió el Pueblo de Chocompta, tan grande, que competia en gentio con el de Turmeque. Era Eminentissimo Theologo; y por esso siempre consultado Señor D. Fr. Juan de los Barrios, a quien asistió en las Constituciones Synodales. Estas buenas prendas de Docto, con las, que tenia de Religioso muy observante, le pusieron en el Provincialato.

No fuera Provincia de Orden de Predicadores, si después de aver embiado á los Religiosos á sus Prioratos, y las reducciones de sus Gentiles, no se hubiera instituido en ella vna Casa de Estudios, en que se leyeran las facultades de Artes, y Theologia. Con la Leccion de gramatica, que se avia empezado, y proseguido desde el año de 1563, avia algunos Estudiantes Seculares y Religiosos, que podían entrar á oir estas facultades. Para ello se señalaron por casa de estudios á este Convento del Rosario, y nombraron, por Regente al P. Mro. Alberto Pedrero, por Lector de Artes al P. Juan de la Drada, y de Theologia al Presentado Fr. Luis López. Este religioso, según parece de vn papel suyo, en que manifiesta sus Grandes Letras vino a esta provincia, de la de México. Tuvo grande opinión de Docto en aquellos tiempos, que dize el P. Fr. Pedro Simón: Estava en la Ciudad de Santo Fé el Padre Fr. Luis López de la Orden de Nuestro Padre Santo Domingo, doctissimo Theologo, con quien en aquellos tiempos se consultaba todo lo que se ofrecia en orden á la reducción de los Indios de este Reyno á nuestra Fé Catholica. Por los años de 1588. Que escribió una Summa de casos de conciencia. 
Esta es vna de las primeras, que se han escrito en estas Indias; y en sus materias le citan todos los que han escrito después. Bolvió á su Provincia de Castilla, y en dos tomos la imprimió el año de 1592.

Celebró mucho Don Gonçalo Ximenez de Quesada, por manifestar el gozo, de ver estudios en la Cabeza del Reyno, que avia conquistado: empezó desde este año a celebrar la fiesta de Santo Thomás, y después donó á este Convento toda la Librería, y fundó una Capellanía, que hasta oy sirve. (...)

A todos los que quisieren ver los libros antiguos de este Convento del Rosario, constará, que desde el año 1563. Hubo en él Leccion de Gramática, á que acudían los hijos de los Conquistadores, y Pobladores de este Reyno. Constará también, que desde el año de 1571. Tuvo Estudios públicos de Artes, y Theología, y las diligencias que hizo (según consta de la Cedula referida) para que se fundára Vniversidad publica, en que se leyeran todas las facultades, pidiendo á su Magestad que dotára la Cathedras. (Zamora, 1945, p. 229 y ss.)

Con esta fuente primaria se evidencia el comienzo de los Estudios Generales en el Convento dominicano del Rosario de Santafé de Bogotá. También se muestra en ella el deseo de fundar universidad pública o estatal, aunque esto último en ningún momento llegara a suceder con la universidad de la Orden de Predicadores. Sin embargo, en Santafé, esta no fue la única orden religiosa en querer establecer universidad. Pues es bien conocida la disputa entre la Universidad Javeriana de los Jesuitas, y la Universidad Tomística de los Dominicos por la concesión de títulos a partir del siglo XVI, pero siendo ambas universidades de órdenes religiosas, y recordando también que hubo otras como la de los agustinos (Egido, 2004, p. 215).

Por otra parte, también es conocida la controversia entre el Colegio de Nuestra señora del Rosario de Bogotá y la Universidad 
Tomística por establecer en cuál institución se empezó a dictar las materias propias de las facultades, y con las que se podría afirmar que ya hay una institución universitaria, controversia histórica que aún continúa. Para esto, se tomará el caso de la Facultad de Medicina. Se afirma que el primer intento por impartir la cátedra de medicina se concretó en el Colegio de San Bartolomé, regido por los Jesuitas en 1636 (Quevedo, 2002, p. 6), dictada por Rodrigo Enríquez de Andrade. Quien arribó a Bogotá con el séquito de fray Cristóbal de Torres de la Orden de Predicadores. Este último llegó para ocuparse del arzobispado de Santafé de Bogotá. Fray Cristóbal fundaría luego una institución educativa de nivel universitario conocida como el Colegio Mayor del Rosario. Este colegio no tuvo ninguna filiación religiosa, aun cuando los dominicos la reclamasen para sí, por haber sido fundada por uno de los suyos. Sin embargo, siempre se conservó como una institución virreinal, esto es, estatal. A diferencia del Colegio de San Bartolomé que perteneció a los jesuitas, hasta el momento de su expulsión en 1767.

Y también se puede afirmar que el primer graduado en medicina, o por lo menos egresado del Colegio Mayor del Rosario, se debió una gestión que se realizó entre 1713 y 1799.

En un informe que sobre la historia de la Cátedra de Medicina del Rosario escribió don Juan Antonio Buenaventura [...], padre rector de la Universidad de Santo Tomás, y que envía al Virrey Mendinueta, a petición de éste, el 25 de abril de 1799, se menciona que a don José de la Cruz se le confirió el título de doctor en esa universidad hacia 1713, a instancias del ilustre ayuntamiento de la capital con el destino de servir a la Cátedra de Medicina del Colegio del Rosario. Sin embargo, el virrey Mendinueta, al recibir el citado informe, lo devuelve al padre rector par que agregue los documentos que cita y, además, solicita informe al rector del Colegio del Rosario. (Emilio Quevedo, 2002, pp. 21-22) 
De esta cita se puede inferir lo siguiente. Por un lado, que efectivamente se dictaron clases de medicina en el colegio del Rosario y, también, que el título lo concedió la Universidad Tomística. Entonces, se puede decir que los estudios universitarios en el siglo XVIII en Santafé de Bogotá no se restringían a una institución en particular, sino al entramado de los colegios y universidades. En el caso de Bogotá, estas últimas eran las que concedían títulos, y que, además, eran universidades pertenecientes a órdenes religiosas. Aunque también hay que tener en cuenta que la Universidad Tomística de los dominicos a partir de 1767 quedó sola en Santafé expidiendo títulos debido a la expulsión de los jesuitas a partir de esta fecha.

En conclusión, se puede afirmar que la Universidad Tomística de Santa Fe de Bogotá en el periodo Hispánico corresponde más a una universidad particular, puesto que estuvo regentada por una orden religiosa. Pero que pudo haber correspondido al título de universidad General, al impartir título de medicina como ocurrió en 1799. Sin embargo, no dejaba de ser particular por no ser una universidad estatal, si es que nos atenemos a la definición de José Abel Salazar acerca de las universidades de Estudios Generales y las de Estudios Particulares.

\section{Referencias}

Belda Plans, J. (2000). La Escuela de Samanca. Madrid: Biblioteca de Autores Cristiano.

Calderón, M. T. (2010). La majestad de los pueblos en la Nueva Granaday Venezuela 1780-1832. Bogotá: Taurus.

Egido, T. (2004). Los jesuitas en España y en el mundo hispánico. Madrid: Marcial Pons, Ediciones de Historia. 
Emilio Quevedo V., G. D. (2002). Historia de la Cátedra de Medicina en el Colegiuo Mayor del Rosario durante la Colonia y la República 1553-1865. Bogotá: Centro Editorial Universidad del Rosario.

Hernádez de Alba, G. (1969). Documentos para la historia de la educación el Colombia. Tomo I. 1540-1653. Bogotá: Patronato colombiano de artes y ciencias.

Plata Quezada, W. E. (2012). Vida y muerte de un convento. Dominicos y sociedad en Santafé de Bogotá (Colombia) Siglos XVI-XVII. Salamanca: San Esteban.

Ramírez González, C. I. (2001). Grupos de poder clerical en las universidades hisánicas I. México: Centro de Estudios sobre la Universidad. Univesidad Nacional Autónoma de México.

Rodríguez Cruz, A. M. (1973). Historia de las Universidades Hispanoamericanas. Periodo hispánico. Tomo I. Bogotá: Instituto Caro y Cuervo.

Zamora, F. A. (1945). Historia de la provincia de san Antonino del Nuevo Reino de Granada. Bogotá: Editorial A. B. G. 


\section{Sobre los autores}

\section{Édgar Arturo Ramírez Barreto}

Docente de tiempo completo del Departamento de Humanidades y Formación Integral, de la Universidad Santo Tomás. Ha sido profesor de Teoría Estética en la Universidad de Bogotá Jorge Tadeo Lozano. Es magíster en Historia y Teoría del Arte, la Arquitectura y la Ciudad de la Universidad Nacional de Colombia y sus áreas de investigación abarcan historia, política, arte, estética y filosofía.

Orcid: https:/ /orcid.org/0000-0003-2082-1538

\section{Miguel Olimpo Moreno Lugo}

Profesor del Departamento de Humanidades y Formación Integral de la Universidad Santo Tomás de Bogotá; tiene estudios de licenciatura en Filosofía y Letras y maestría en Filosofía Latinoamericana. En la actualidad realiza estudios de Doctorado en Filosofía y su trabajo investigativo gira en torno a la filosofía de Tomás de Aquino en la vertiente de Leonardo Polo.

Orcid: https://orcid.org/0000-0002-1523-8965 
Francisco Javier Yate Rodríguez

Licenciado en Filosofia y Letras, y magíster en Filosofia Latinoamericana de la Universidad Santo Tomás. Docente del Departamento de Humanidades y Formación Integral de la Universidad Santo Tomás en espacios académicos como: Filosofía Institucional y Antropología. Se ha desempeñado como líder de las Cátedras Opcionales del Departamento de Humanidades y Formación Integral de la Universidad Santo Tomás.

Orcid: https://orcid.org/0000-0002-6277-2458

Juan Sebastián Ballén Rodríguez

Licenciado en Filosofía y Lengua Castellana de la Universidad Santo Tomás (Bogotá). Magíster en Filosofía por la Pontificia Universidad Javeriana (Bogotá). Es profesor universitario y miembro ordinario del Círculo Latinoamericano de Fenomenología (Clafén). Se ha desempeñado como investigador en diversos campos de la filosofia, tales como la estética, la filosofia del arte, la antropología filosófica, la fenomenología y la hermenéutica, la historia de la ciencia y la filosofia colonial en Colombia. Cursa actualmente el Doctorado en Filosofía en la Pontificia Universidad Javeriana.

Orcid: https://orcid.org/0000-0001-5214-3108 




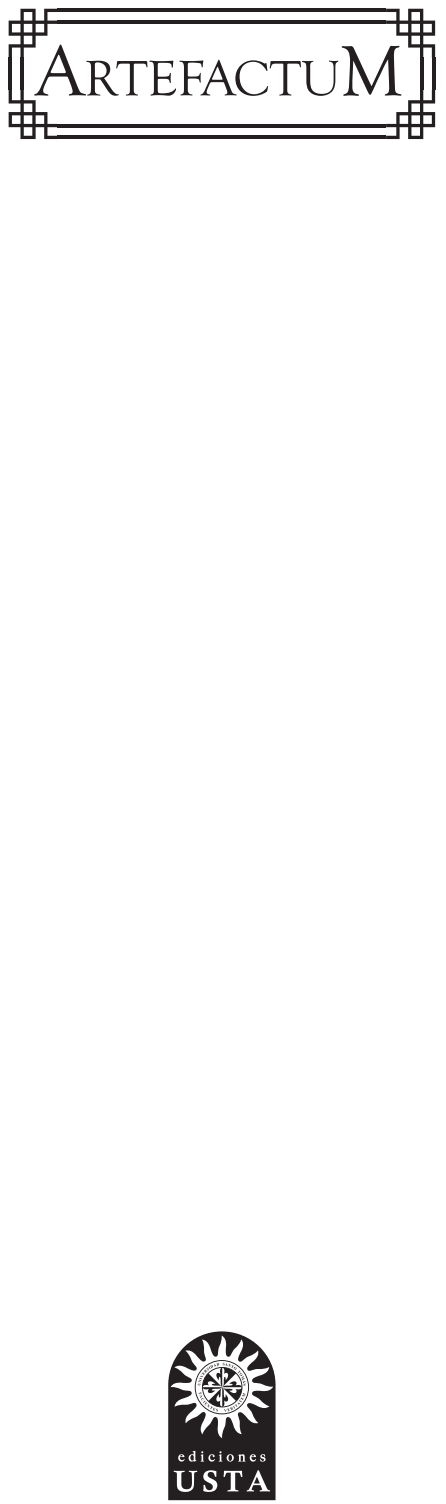

Esta obra se editó en Ediciones USTA.

Se usó papel propalcote de 300 gramos para la carátula y papel bond beige de 75 gramos para páginas internas.

Tipografías de las familias Goudy Old Style BT y Baskerville.

2019 
Oon este libro se quiere mostrar, a través de diversas investigaciones, lo que significó la expresión Estudios Generales dentro del contexto universitario y, específicamente, en el de la Orden de Predicadores durante el periodo hispánico de Santa Fe de Bogotá, la Nueva Granada y en el ámbito educativo de España y Europa; toda vez que este modelo educativo nace en los mismos comienzos de la Orden a mediados del siglo xiII. 